\title{
Aprender estructuras aritméticas a través de la interdisciplinariedad con Ciencias Naturales ${ }^{1}$
}

Artículo de investigación

Fecha de Recepción: 3 abril 2018.

Fecha de Aprobación: 4 agosto 2018.

Eliana Marcela Figueredo Piragauta* Maritza Figueredo Piragauta ** Clara Emilse Rojas Morales***

\section{Resumen}

Se propuso una estrategia didáctica articulada basada en talleres con diversas situaciones problema, empleando contenidos relacionados con el componente del entorno vivo de las Ciencias Naturales, y haciendo uso de la indagación. Se asumen referentes desde las Matemáticas y las Ciencias Naturales, que aportan elementos para transformar las prácticas pedagógicas de estas asignaturas en el aula. Se aplicó una metodología de investigación acción educativa desde la postura de Kemmis (1988), implementando cuatro fases: diagnóstico, diseño, aplicación, resultados y productos. El enfoque es cualitativo; la técnica empleada fue la observación; y los instrumentos aplicados, el diario de campo, los talleres y las grabaciones. La implementación de la estrategia mejoró la construcción y apropiación de conocimiento alrededor de las estructuras aritméticas, el afianzamiento de competencias de comunicación, razonamiento y resolución de problemas en Matemáticas y la indagación, uso comprensivo del pensamiento científico y explicación de fenómenos en Ciencias Naturales, facilitando la construcción de aprendizajes significativos pertinentes para comprender y enfrentar las situaciones de la vida real.

Palabras clave: pensamiento, estructuras aritméticas, estrategia de enseñanza, aprendizaje activo, Ciencias Naturales.
1 El artículo presenta reflexiones sobre la investigación "Articulación entre Matemáticas y Ciencias Naturales: una estrategia para aprender Estructuras Aritméticas" realizada en la Institución Educativa La Independencia del municipio de Sogamoso, Boyacá, con estudiantes de grado tercero.

* Institución Educativa

La Independencia, Boyacá-Colombia elmafipi@yahoo.es *** Institución Educativa

La Independencia,

Boyacá-Colombia marifipi2006@yahoo.es

*** Universidad Pedagógica y Tecnológica de Colombia, Boyacá-Colombia

clara.rojas@uptc.edu.co

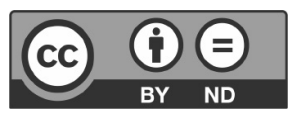




\section{Introducción}

Uno de los objetivos de la Educación Matemática debe ser, sin duda, captar el interés de los estudiantes y motivar su propia vivencia de las Matemáticas. El objetivo de las Ciencias Naturales y Educación Ambiental, es el desarrollo científico, tecnológico, ético y socioafectivo, el cual incluye procesos dinámicos que hacen parte de la interacción entre el ser vivo y su entorno. El pensamiento científico y el matemático se relacionan naturalmente entre sí, permitiendo avanzar hacia un saber hacer flexible, que articula conocimientos de estas asignaturas, es por esta razón que los docentes deben buscar estrategias de enseñanza transversales que motiven, cautiven y desarrollen competencias en estas áreas.

El artículo hace parte del proyecto de investigación titulado "Articulación entre Matemáticas y Ciencias Naturales: una estrategia para aprender estructuras aritméticas", que tiene como objetivo: establecer una estrategia didáctica que articule las Matemáticas con las Ciencias Naturales y afiance el aprendizaje de estructuras aritméticas en los niños de grado tercero de primaria. La metodología está enmarcada en la investigación acción educativa (desde la postura de Kemmis) con enfoque cualitativo.

Se busca reforzar la enseñanza de las estructuras aritméticas, a través del razonamiento y resolución de situaciones problema, en un grupo conformado por 18 estudiantes de grado tercero de la Institución Educativa La
Independencia (IELI) de Sogamoso; además, se analiza el papel del docente y el impacto en el mejoramiento de las prácticas pedagógicas de aula como herramienta para favorecer los procesos de enseñanza y aprendizaje activo de los contenidos curriculares de las áreas de Matemáticas y Ciencias Naturales.

Para el planteamiento del problema, se parte del análisis de los resultados de las pruebas Saber y el Índice Sintético de Calidad Educativa (ISCE) del Ministerio de Educación Nacional (MEN), de los estudiantes de los grados tercero y quinto de la IELI en las áreas de Matemáticas y Ciencias Naturales de los años 2014 y 2015, donde se evidencia dificultades en las competencias de razonamiento y resolución de problemas, y debilidad en el componente ciencia, tecnología y sociedad. Es por esta razón que, surge la inquietud de las docentes que orientan estas asignaturas, de diseñar y aplicar una estrategia interdisciplinar que promueva la construcción del conocimiento de dichas áreas.

Trasla aplicación dela prueba diagnóstica diseñada en conjunto por las docentes maestrantes, se evidencia dificultad en la ubicación de cantidades según su valor posicional al momento de realizar operaciones de adición y sustracción; así como al efectuar clasificación empleando las relaciones de orden en forma ascendente y descendente. Desde el punto de vista semántico, se reconoce desconocimiento de términos propios del lenguaje matemático manifestándose en problemas de comprensión lectora. Estas debilidades deben ser tratadas desde un punto de vista pedagógico y 
didáctico, para evitar que los estudiantes, al continuar su ciclo escolar, presenten bajo desempeño académico, poco interés, apatía, reprobación de grados y deserción.

Las docentes, al diseñar e implementar la estrategia didáctica, pretenden superar las falencias que revelan la mayoría de los estudiantes del grado tercero en procesos como: interpretación y resolución de enunciados verbales de problemas aditivos y multiplicativos, para ello elaboran talleres empleando la interdisciplinariedad con la inclusión de contenidos del entorno vivo (los seres vivos, clasificación, especies amenazadas por la extinción, el reino animal, clasificación -vertebrados e invertebrados-), el cuerpo humano y sus sistemas (circulatorio y el corazón, el sentido del oído), los componentes de los talleres son: contexto significativo con las Ciencias Naturales, conexión con Matemáticas, aprendo en contexto resolviendo problemas y pongo a prueba mis capacidades en donde se incluyen actividades lúdicas en el uso de los algoritmos de las operaciones, que motivan y llaman la atención de los estudiantes, como lo son: rompecabezas, mategramas, figuras de animales para colorear. De esta forma, se contribuye a la innovación y cambio de didáctica en la enseñanza de estas asignaturas.

Tras la revisión documental tanto de libros, informes y tesis referentes al tema de la investigación, es relevante resaltar el trabajo de Flores, Castro y Fernández (2015) quienes afirman que es importante que los docentes de Educación Primaria sepan, entiendan y utilicen conocimientos necesarios para diseñar tareas escolares y unidades didácticas de Matemáticas, que faciliten el aprendizaje y sean acordes con los intereses y necesidades de los estudiantes; además, estos autores trabajan sobre aspectos didácticos de la enseñanza de las estructuras aritméticas en los escolares de Primaria a través de situaciones cotidianas y reafirman el desarrollo gradual de estas estructuras durante los primeros ciclos de la educación básica, haciendo énfasis en la resolución de problemas que involucren situaciones de añadir, reunir, quitar, separar y comparar. Vergnaud (1986) afirma al respecto, que:

La mayor parte del trabajo que se hace en la escuela dedicado al significado de las operaciones se ha limitado a resolver problemas verbales sin sentido para el estudiante ya que generalmente se les enseña un solo tipo de situación; como por ejemplo, ver la suma como reunir o juntar, así como al momento de abordar el significado de la resta, interpretándose ésta como la acción de quitar o sobrar, sin tener presente la variedad de situaciones en las que subyacen estas operaciones aritméticas, de igual forma esta dificultad también es evidente en el aprendizaje de la estructura multiplicativa (p.62).

Por consiguiente, la etapa infantil es de enorme trascendencia para la educación matemática posterior del niño, ya que es en esta etapa donde se forman los conceptos básicos o primarios y las bases sobre las que, posteriormente, se construirá todo el aprendizaje. Si estas bases elementales están mal cimentadas
La mayor parte del trabajo que se hace en la escuela dedicado al significado de las operaciones se ha limitado a resolver problemas verbales sin sentido para el estudiante ya que generalmente se les enseña un solo tipo de situación 
o son frágiles, pueden impedir o dificultar el aprendizaje posterior. En la educación primaria, el estudiante ha de ser encauzado para que evolucione cada vez hacia procesos más abstractos de pensamiento.

Por esta razón, la enseñanza de las Matemáticas en general y lo que hace referencia a la enseñanza y aprendizaje de estructuras aritméticas, debe centrar sus esfuerzos primero, en los conocimientos y modelos mentales que poseen los maestros de Básica Primaria para generar reflexiones y transformaciones profundas que les permita mejorar sus prácticas de aula $y$, por ende, el desempeño, el desarrollo de competencias y la construcción de verdaderos aprendizajes significativos en los niños.

\section{Marco teórico}

A continuación, se describen algunos referentes teóricos que fueron relevantes en la investigación.

\section{Enseñanza de las Matemáticas}

En este sentido, es importante reconocer que la Matemática es una área básica que potencia el pensamiento del ser, para desarrollarlo en su entorno y en su problemática diaria (planteamiento y solución de problemas), y contribuye en la formación de la persona para hacerla competente en la vida laboral. Por tal motivo, la enseñanza de las Matemáticas no solo depende de que los estudiantes manejen conceptos y procedimientos matemáticos, sino que además incrementen ampliamente un pensamiento matemático, de manera que puedan explorar la realidad, representarla, explicarla, y actuar en ella.

\section{Pensamiento matemático}

A mediados del siglo XX, Piaget (1978), en sus estudios de psicología, asume el pensamientocomo la acción de formar, relacionar ideas y conceptos, y el pensamiento humano es conocido como una de las funciones mentales superiores, donde el razonamiento, la memoria, la abstracción y los demás procesos mentales, son asumidos $\mathrm{y}$ estudiados por esta misma ciencia. Los matemáticos definen el pensamiento matemático como el proceso de construcción de los conceptos y los procesos mentales, este se desarrolla para mostrar la acción matemática como una forma especial de la actividad humana, el interés por desarrollarlo es caracterizar o modelar los procesos de comprensión de los conceptos y procesos matemáticos.

Los fundamentos del pensamiento matemático están presentes desde edades muy tempranas en los niños, como consecuencia de los procesos de desarrollo y de las experiencias que viven al interactuar con el contexto.

\section{Pensamiento numérico}

En la investigación, se prioriza el pensamiento numérico y el proceso general de las matemáticas que se refiere a la formulación y resolución de problemas; puesto que, en el primero, se aborda el significado de las operaciones por medio del estudio de las estructuras aritméticas, y en el segundo se da tratamiento a la 
formulación y resolución de problemas; específicamente, los problemas verbales de tipo aditivo y multiplicativo donde intervienen las operaciones de suma, resta, multiplicación y división, los cuales se desarrollan en la educación básica primaria.

\section{Rol del profesor en la enseñanza del pensamiento matemático}

El desafío de quien ejerce la profesión docente, es crear condiciones para que puedan darse en las instituciones educativas procesos de aprendizaje, de innovación y formación diseñados por los mismos docentes, de ambientes de aprendizaje que también permitan a los profesores aprender y a los colegios mejorar dichos procesos.

Un aspecto que propone el MEN (1998b), es la transformación de la preponderancia del conocimiento fragmentado, en contraste con un conocimiento que promueve el desarrollo del pensamiento matemático. Este cambio significa para el docente una gran responsabilidad, pues debe hacer de la enseñanza de las matemáticas, una herramienta para hacer efectivo en los estudiantes el desarrollo de la capacidad de razonamiento, el estímulo del uso de esquemas y representaciones gráficas, además de promover el trabajo cooperativo y lograr que intervengan en la construcción de su propio conocimiento.

Esta transformación en la enseñanza de las Matemáticas, debe permitir que el estudiante formule, pruebe, construya modelos, lenguajes, conceptos, estructuras. Para lograrlo, debe intercambiar con otros y reconocer los conocimientos que son fruto de la experiencia cultural de la sociedad.

\section{Enseñanza de las Ciencias \\ Naturales y Educación Ambiental}

Ahora bien, las Ciencias Naturales y Educación Ambiental es entendida como una área básica del conocimiento, que promueve el desarrollo científico, tecnológico, ético y socioafectivo, que se encuentra en constante evolución y permite acceder a las innovaciones de la globalización; incluye procesos dinámicos que hacen parte de la interacción entre el ser vivo $\mathrm{y}$ su entorno, haciendo posible el avance en los procesos científicos que ayudan al logro de calidad de vida en todos los ámbitos, contribuye al desarrollo integral de los estudiantes que asumen una posición de reflexión, análisis crítico y conocimiento del entorno a través de la construcción del pensamiento y de la acción en el tratamiento de personas, además de resaltar el valor de la vida y la importancia de la armonía con la naturaleza.

Al trabajar esta asignatura de manera interdisciplinar con las Matemáticas, los docentes deben conocer los contenidos curriculares, buscar temáticas afines en los planes de área y definir los conceptos y actividades que se van a desarrollar en el diseño y planificación colectiva de talleres que desarrollen competencias en las dos asignaturas.

\section{Pensamiento científico}

El Ministerio de Educación Nacional (1998a) asevera que:
El desafío de quien ejerce la profesión docente, es crear condiciones para que puedan darse en las instituciones educativas procesos de aprendizaje 


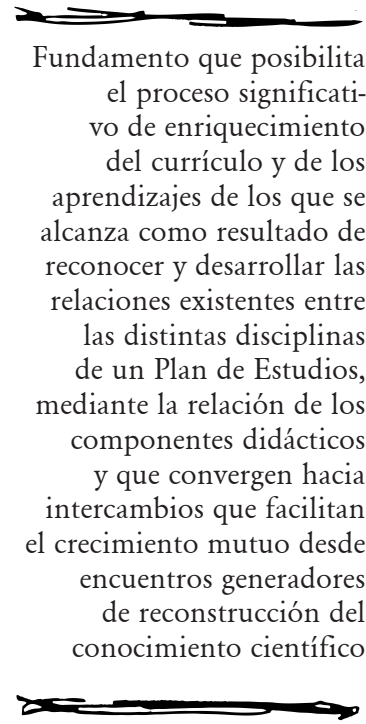

El estudiante debe desarrollar un pensamiento científico que le permita contar con una teoría integral del mundo natural, dentro del contexto de un proceso de desarrollo humano equitativo y sostenible, que le proporcione una concepción de sí mismo y de sus relaciones con la sociedad y la naturaleza armónica con la preservación de la vida en el planeta mediante la formación científica y la motivación para investigar, plantear preguntas, criticar, reflexionar, saber, ubicar, relacionar, analizar y sintetizar información. Se concluye que la educación en ciencias tiene como finalidad central el desarrollo del pensamiento científico, como herramienta clave para desempeñarse con éxito en un mundo impregnado por la ciencia (p. 60).

\section{Rol del educador en la enseñanza de las Ciencias Naturales}

El profesor de Ciencias Naturales y Educación Ambiental, debe enseñar para la construcción permanente de valores adecuados a las necesidades actuales para una mejor sociedad en términos de calidad de vida. La enseñanza de esta debe enfatizar en la construcción de procesos, más que en los métodos de transmisión de resultados; y debe explicitar las relaciones y los impactos de la ciencia y la tecnología en la vida del hombre, su entorno natural y la sociedad (VázquezAlonso \& Rodríguez-Cruz, 2014). La calidad de la enseñanza de las Ciencias Naturales se ve ampliamente favorecida con el compromiso real del profesor, la implementación de didácticas innovadoras haciendo uso del entorno natural para desarrollar competencias de indagación, uso comprensivo del conocimiento científico y explicación de fenómenos.

\section{La interdisciplinariedad}

La interdisciplinariedad sirve como estrategia para una mayor fluidez entre el trabajo teórico y el práctico. No es una receta, ni es una directiva, es un proceso, puesto que se fomenta $y$ perfecciona paulatinamente, durante la propia actividad práctica.

Según Addine (2002),es el

Fundamento que posibilita el proceso significativo de enriquecimiento del currículo y de los aprendizajes de los que se alcanza como resultado de reconocer y desarrollar las relaciones existentes entre las distintas disciplinas de un Plan de Estudios, mediante la relación de los componentes didácticos y que convergen hacia intercambios que facilitan el crecimiento mutuo desde encuentros generadores de reconstrucción del conocimiento científico (p. 21).

La interdisciplinariedad es un proceso y una filosofía de trabajo, Fiallo (2012) afirma que es una forma de pensar y de proceder para conocer la complejidad de la realidad objetiva y resolver cualquiera de los complejos problemas de este planeta. Es cuando hay cooperación entre varias disciplinas e interacciones que conllevan a enriquecimientos mutuos. Estas interrelaciones van desde la simple comunicación de ideas hasta la integración común de contenidos, metodologías y estrategias didácticas al desarrollar las actividades. 
Problemática de la enseñanza de las Ciencias Naturales y las Matemáticas

Valverde y Näslund-Hadley (2010) hacen referencia a los problemas que presentan los estudiantes en América Latina y el Caribe en cuanto a la formación en Matemáticas y Ciencias Naturales en los niveles de preescolar, primaria y secundaria. Estos autores afirman que los estudiantes no están siendo debidamente preparados en estas asignaturas para enfrentar los cambios de una sociedad cada vez más interconectada, debido a la falta de destreza de los docentes, programas débiles, materiales de enseñanza inadecuados y a la mecanización y memorización de contenidos en las aulas.

Este estudio permite realizar una reflexión sobre los resultados de los estudiantes de la región en evaluaciones internacionales del rendimiento de la educación, ya que se encuentran por debajo de los países pertenecientes a Asia oriental y de los países industrializados asociados a la Organización para la Cooperación y el desarrollo Económico (OCDE). Por estos argumentos, es importante el trabajo interdisciplinar de dichas asignaturas enmarcado en la investigación, ya que propende por transformar las prácticas de aula en las Matemáticas y las Ciencias Naturales.

Enseñanza y aprendizaje de las estructuras aritméticas

Para la conceptualización de la enseñanza y aprendizaje de las estructuras aritméticas en esta investigación, se han tenido como referentes importantes autores como Castro, Rico y Castro (1995) y Flores et al. (2015), quienes a través de rigurosas investigaciones y libros derivados de las mismas, han logrado construir y consolidar propuestas relacionadas con el estudio de las estructuras aritméticas, los cuales se referencian como soporte teórico.

Flores et al. (2015) consideran que, en las matemáticas escolares, se denomina estructura aritmética a un conjunto de números en los que hay definidas operaciones aritméticas ligadas por una relación de reciprocidad, operaciones que verifican unas propiedades, y un campo de problemas que se abordan y resuelven mediante las mismas. En la aritmética de los números naturales, destacan dos estructuras:

Estructura aditiva: operaciones de suma y resta con números naturales, sus propiedades y el campo de problemas que resuelven.

Estructura multiplicativa: operaciones de producto y división con números naturales, sus propiedades y el campo de problemas que resuelven.

Los docentes de Educación Básica primaria deben conocer y abordar las diferentes etapas de aprendizaje de las operaciones, ya que son fundamentales en el proceso de construcción del conocimiento en la enseñanza sobre estructuras aritméticas.

Etapas en el aprendizaje de las operaciones

En el proceso de aprendizaje de las operaciones, se distinguen varias 
etapas que, a su vez, comprende dos fases: una conceptual, cuyo propósito es familiarizar a los niños con el concepto de la operación; y otra procedimental, basada en desarrollar sus estrategias y hábitos de cálculo. Las dos están encaminadas a crear y afianzar herramientas para resolver problemas.
La fase conceptual comprende la actuación directa, con elementos concretos (acciones), el uso de modelos y la simbolización. La fase procedimental o de cálculo se concentra en el aprendizaje y empleo de hechos numéricos aislados y formando tablas, la creación y memorización de algoritmos y su utilización en la resolución de problemas.

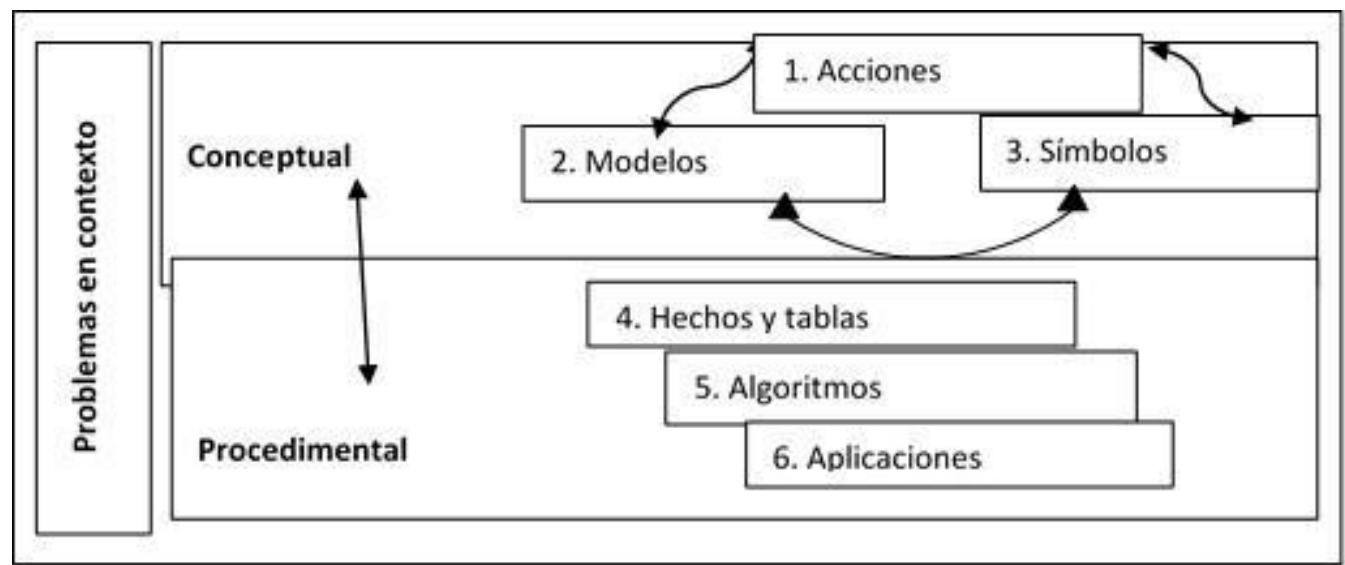

Figura 1. Esquema de la enseñanza y aprendizaje de las operaciones aritméticas.

Fuente: Flores et al.,2015, p.206.

Enseñanza y aprendizaje de la Estructura Aditiva

La estructura aditiva, de la que la suma y la resta son sus representaciones más sencillas, subyace, según Carpenter, Moser y Romberg (1982), en gran número de conceptos matemáticos, y su desarrollo en el niño ocupa un extenso período de tiempo ya que ha de cubrir la transición desde los recuentos informales y las estrategias propias que los niños realizan al margen de su instrucción, hasta el uso de datos numéricos memorizados y los algoritmos formales de la adición y sustracción. Este es un período crítico para el aprendizaje de las matemáticas por los niños y se creé que algunas de las dificultades posteriores en matemáticas tienen su origen en la deficiente instrucción inicial de la suma y la resta.

Según Piaget (1978), los conceptos más elementales del número no están completamente desarrollados en los niños antes de los 7 años de edad, aun cuando los conceptos de adición y sustracción, que suponen conocimientos de conceptos numéricos básicos, empiecen a la edad de 6 años. Muy pronto, los niños entienden que 
la secuencia numérica se puede utilizar para realizar operaciones aritméticas.

Vergnaud(1988) expresa que la estructura aditiva es "el conjunto de situaciones cuyo tratamiento implica una o varias adiciones o sustracciones, y el conjunto de los conceptos y teoremas que permiten analizar esa situaciones como tareas matemáticas” (p.147). Además, dice que "las relaciones aditivas son relaciones ternarias, que se encadenan de diversas maneras ofreciendo gran variedad de estructuras aditivas" (p.147).

Para efectos de esta investigación, se retoma la clasificación sobre los problemas aditivos simples realizada por Castro et al. (1995), la cual se centra en el lugar de la incógnita en los problemas simbólicos.

\section{Tabla 1}

Clasificación de los problemas Aditivos Simples

\begin{tabular}{ll}
\multicolumn{2}{l}{ Tipos de sentencias abiertas } \\
\hline Para la suma & Para la resta \\
\hline $\mathrm{a}+\mathrm{b}=?$ & $\mathrm{a}-\mathrm{b}=?$ \\
\hline $\mathrm{a}+?=\mathrm{c}$ & $\mathrm{a}-?=\mathrm{c}$ \\
\hline$?+\mathrm{b}=\mathrm{c}$ & $?-\mathrm{b}=\mathrm{c}$ \\
\hline$?=\mathrm{a}+\mathrm{b}$ & $?=\mathrm{a}-\mathrm{b}$ \\
\hline $\mathrm{c}=?+\mathrm{b}$ & $\mathrm{c}=?-\mathrm{b}$ \\
\hline $\mathrm{c}=\mathrm{a}+?$ & $\mathrm{c}=\mathrm{a}-?$
\end{tabular}

Fuente: Castro et al.,1995, p.37.

Para abordar la elaboración de la estrategia para aprender las estructuras aditivas, las docentes de la investigación toman como referente teórico los ejemplos de situaciones problema de estructura aditiva condensada en los Lineamientos Curriculares Matemáticas
(1998), que, a su vez, contemplan Castro y Vergnaud.

\section{Enseñanza de la Estructura Multiplicativa}

Se infiere de la interpretación de la postura de Castro et al. (1995), que al igual que en el caso de la adición y la sustracción, el aprendizaje de la multiplicación y la división es el inicio de la construcción mental de una nueva estructura: la estructura multiplicativa, que es una de las más valiosas de la matemática.

Por consiguiente, existen algunas diferencias con respecto a la estructura aditiva. Iniciar a trabajar en la multiplicación y en la división, exige que el estudiante posea uso y dominio de los números, conozca su simbolización. Todo esto, en un grado mayor que en el caso de la adición y la sustracción.

La enseñanza de la adición y la sustracción se trabaja simultáneamente a la adquisición del concepto de número, mientras que la multiplicación y la división son operaciones que requieren un dominio previo de los números y su simbolización. El fundamento de esto se encuentra en el concepto propio de cada operación. Multiplicar es reiterar una cantidad, en su nivel más intuitivo. Los dos términos de la multiplicación responden a contextos diferentes; el multiplicando responde a la cantidad que se repite, siendo este un número cardinal concreto, con objetos que se ven. El multiplicador representa las veces que se repite la cantidad inicial, y es un cardinal de segundo orden, más abstracto que el anterior, y por ende se debe simbolizar 
de inmediato. Exactamente ocurre con la división, dividir es repartir una cantidad en partes iguales; el dividendo hace referencia a la cantidad que se reparte, tratándose de un número en contexto cardinal, expresado en objetos concretos; el divisor es el número de partes en que se divide, un número cardinal más abstracto, que inmediatamente pasa a escribirse simbólicamente. Tanto en una situación como en la otra, se emplean dos niveles diferentes de cardinación, y por esto es necesario usar los signos numéricos propios casi desde el inicio. Por tal complejidad, se suele esperar al segundo año de escolaridad para iniciar el aprendizaje de la estructura multiplicativa, puesto que los estudiantes ya han aprendido y manejan la estructura aditiva.

Existe una segunda razón de tipo práctico. Como la multiplicación es representada como una suma reiterada, conviene poseer un cierto dominio de esta, que permita un cálculo más efectivo en los productos. Para no complicar la multiplicación con dificultades propias de la adición, se acostumbra dejar uno o dos cursos de diferencia entre el estudio de ambas estructuras. De este modo, los algoritmos y destrezas de la adición habrán madurado lo suficiente para permitir un inicio más sólido y seguro en la multiplicación.

\section{Clases de problemas de Estructura Multiplicativa}

Tras el análisis que hace Vergnaud (citado en Castro et al.,1995) de los problemas de multiplicación y división, muestra que los problemas "simples" se sitúan en dos grandes categorías:

\section{Categoría: isomorfismo de medida}

Es una estructura que engloba a los problemas referentes a una proporcionalidad simple directa entre las dos magnitudes trabajadas.

El producto de medida es una estructura que abarca tres magnitudes: M1, M2 y M3, de tal manera que una de ellas, M3, es el producto cartesiano de las otras dos.

\section{$\mathrm{M} 1 \mathrm{X} \mathrm{M} 2=\mathrm{M} 3$}

La categoría 2 es la proporción múltiple, la cual se refiere a problemas de proporcionalidad en los que intervienen al menos tres magnitudes y que son, por tanto, problemas compuestos en los que para su resolución hay que emplear más de una operación.

Al observar la gran variedad de situaciones problema que se deben abordar y resolver, es preocupante que la mayoría de docentes desconocen el lenguaje matemático y solo en el aula de clase trabajan la ejercitación de algoritmos, sin relacionarlos con acciones y problemas contextualizados.

\section{Metodología}

El anterior marco teórico e investigativo, permite a las docentes de la investigación diseñar y aplicar una estrategia didáctica, alejada de la aplicación de algoritmos para sumar, restar, multiplicar y dividir, sino centrada en la comprensión y resolución de tareas matemáticas articuladas en contextos de las Ciencias Naturales, de tal forma que se motiva a los estudiantes y se logra desarrollar 
competencias científicas y matemáticas, y aprendizajes activos con significado.

Se asume una metodología enmarcada en la investigación acción educativa con enfoque cualitativo. La técnica empleada es la observación; y los instrumentos, el diario de campo, los talleres, los cuadernos de los estudiantes y las grabaciones. Se aplica la investigación acción desde la postura de Kemmis (1988), implementando cuatro fases: diagnóstico, diseño, aplicación, resultados y productos (ver figura 2); donde las acciones de planificar (P), actuar (A), observar $(\mathrm{O})$ y reflexionar $(\mathrm{R})$, transforman las prácticas pedagógicas en el aula. La unidad de análisis es el grupo de grado tercero, conformado por 18 estudiantes ( 8 mujeres y 10 hombres) con edades entre los 8 y 9 años.

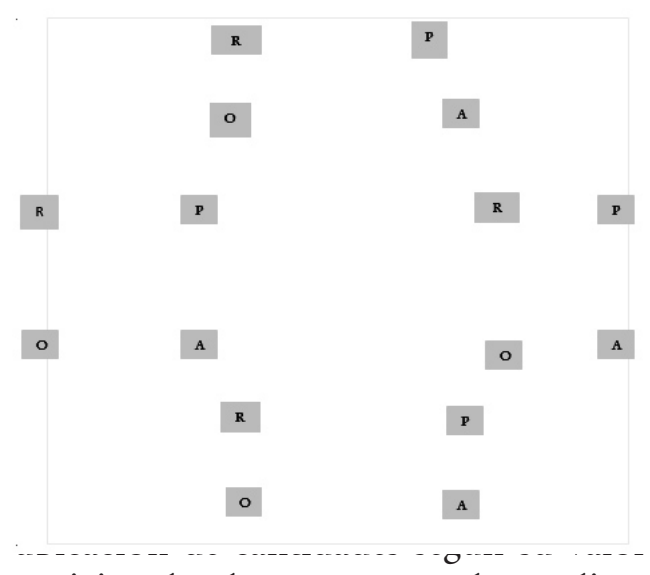

posicional al momento de realizar operaciones de adición y sustracción al solucionar problemas; así como al efectuar clasificación empleando las relaciones de orden en forma ascendente y descendente.

En la segunda fase (Diseño), se llevó a cabo un trabajo de construcción colectiva de las docentes del área de Matemáticas y Ciencias Naturales, en el cual desde la planeación de los contenidos curriculares correspondientes al grado tercero en Ciencias Naturales (entorno vivo) y Matemáticas (estructura aditiva y multiplicativa) y a partir de la revisión documental de los Lineamientos Curriculares, Estándares Básicos de Competencias y Derechos Básicos de Aprendizaje, y teniendo en cuenta la tradición investigativa, se reúnen para el diseño de talleres articulados en su mayoría en situaciones problema con un contexto significativo de contenidos propios del currículo de Ciencias Naturales. Esta estrategia surge puesto que se observa la necesidad de cambiar la manera tradicional de trabajar dichas asignaturas que se venían orientando de forma fragmentada e individual por cada docente, ya que los estudiantes demuestran interés por esta temática (componente entorno vivo) y se cautiva la atención por aprender Matemáticas de una manera menos compleja.

Una vez finalizado el diseño de los talleres, se envían para validación por parte de la asesora de la investigación $y$ de un docente externo para el mejoramiento de la estructura de los talleres, antes de ser aplicados a los estudiantes de grado tercero de la IELI.

\section{Estructura de los talleres}

Se elaboran talleres empleando la interdisciplinariedad con la inclusión de contenidos del entorno vivo y se plantean situaciones problema de estructuras aritméticas a partir de las temáticas sobre: los seres vivos, los
En la segunda fase (Diseño), se llevó a cabo un trabajo de construcción colectiva de las docentes del área de Matemáticas y Ciencias Naturales, en el cual desde la planeación de los contenidos curriculares correspondientes al grado tercero en Ciencias Naturales (entorno vivo) y Matemáticas (estructura aditiva y multiplicativa) y a partir de la revisión documental de los Lineamientos Curriculares, 
animales vertebrados e invertebrados $\mathrm{y}$ algunos sistemas del ser humano. A continuación, se ilustra la estructura de uno de los talleres diseñados y aplicados a los estudiantes de grado tercero de la IELI.
La fase 3 (Aplicación) constituye la puesta en marcha de la propuesta, mediante la aplicación de los talleres diseñados, donde los estudiantes manipulan, exploran material didáctico (del contexto), participan y proponen soluciones desde su nivel de

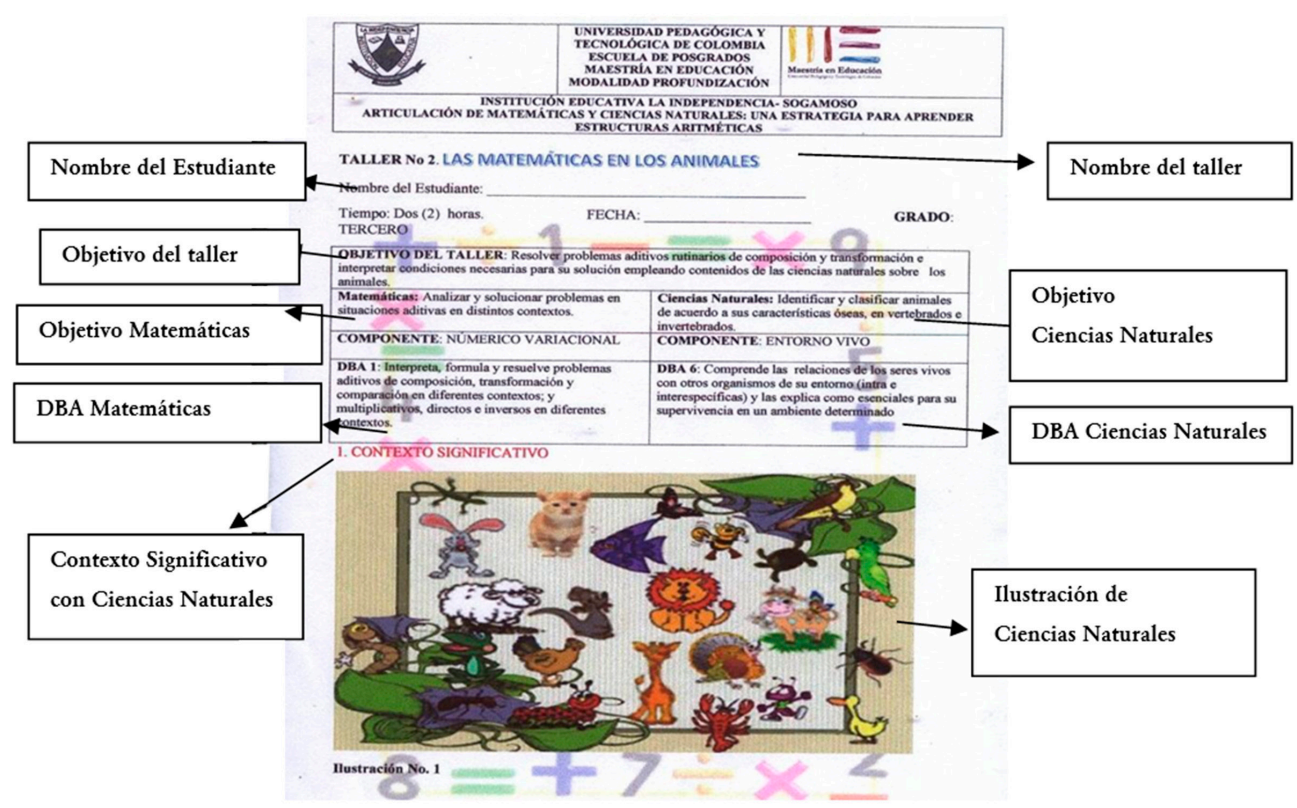

Figura 3. Contexto Significativo con las Ciencias Naturales.

Fuente: Figueredo\& Figueredo, 2017, p. 128.

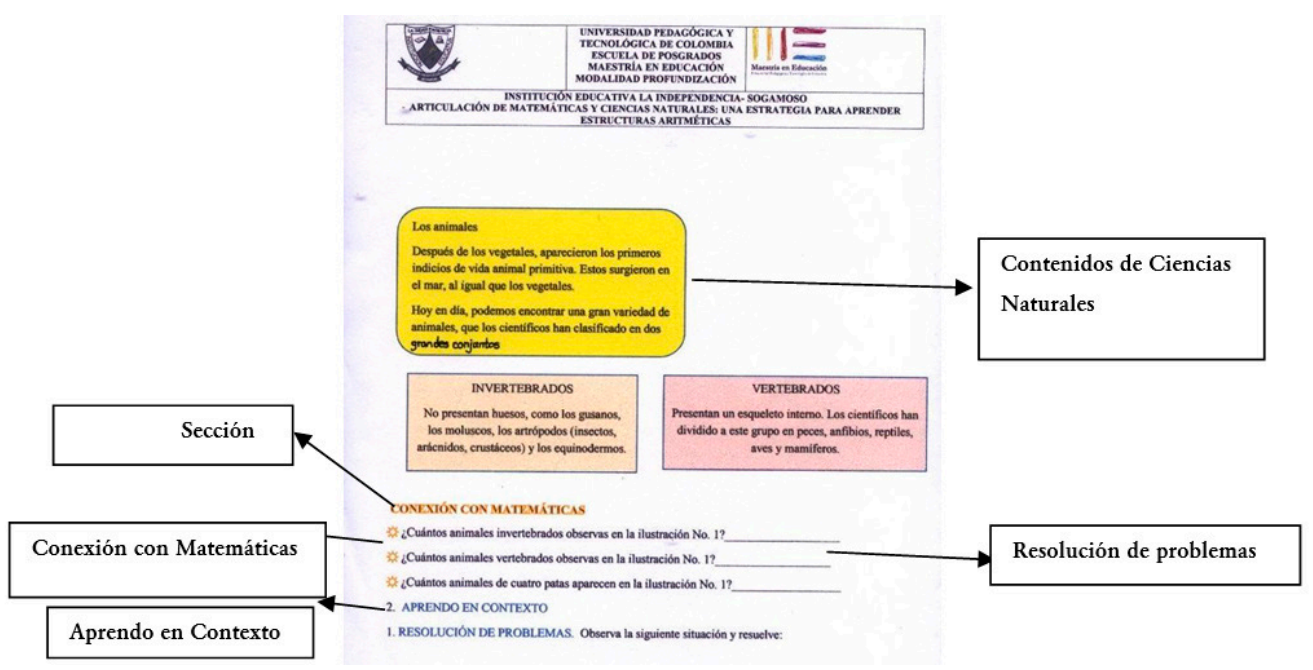

Figura 4. Conexión con Matemáticas.

Fuente: Figueredo\& Figueredo, 2017, p. 129 

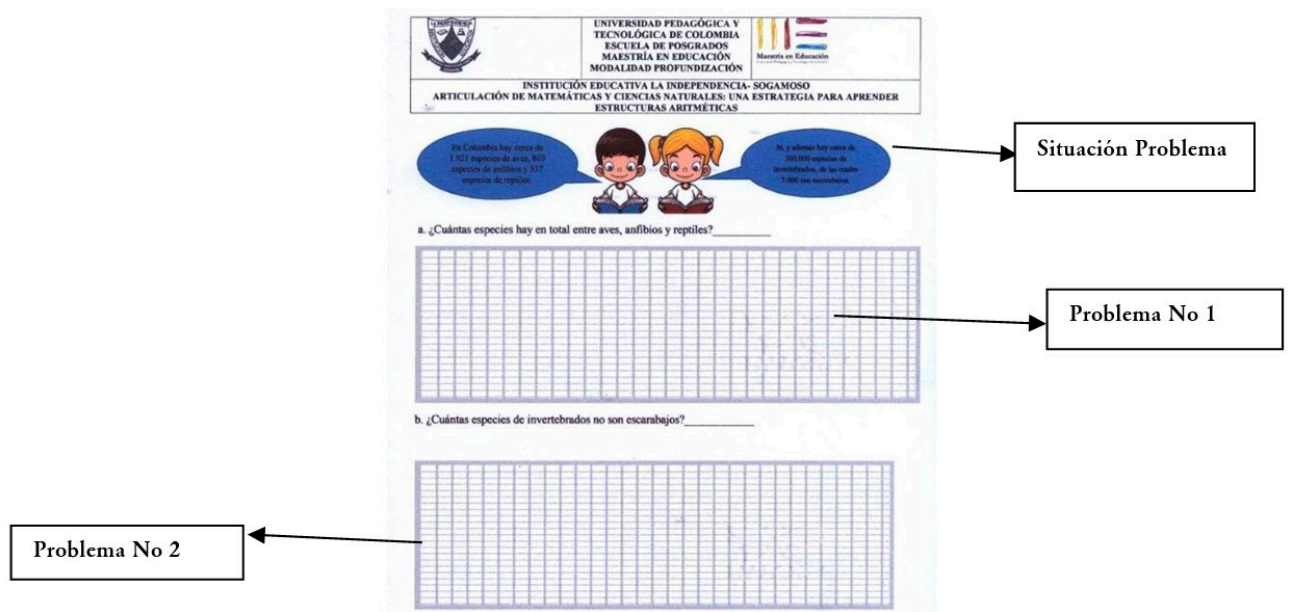

Figura 5. Aprendo en Contexto resolviendo problemas.

Fuente: Figueredo\& Figueredo, 2017, p. 130.

Nombre de la actividad

Pongo a prueba mis capacidades

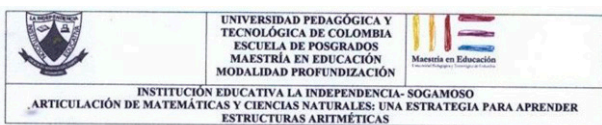

6. Divierte realizando las operaciones y coloreando teniendo en cuenta la clave.

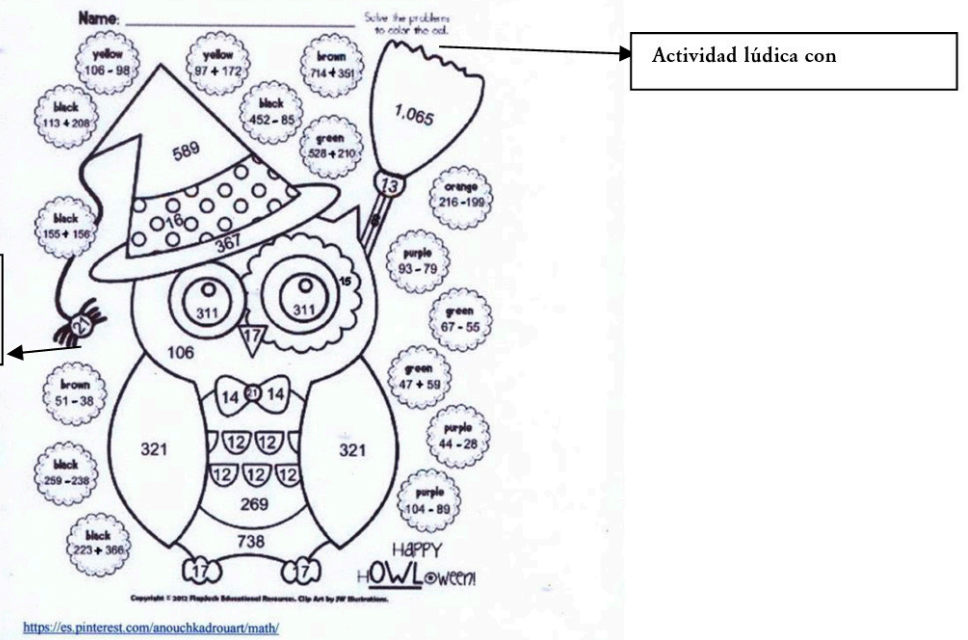

Figura 6.Pongo a prueba mis capacidades.

Fuente: Figueredo\& Figueredo, 2017, p. 139.

comprensión, comparten y socializan respuestas, dudas y procedimientos con sus pares, enriqueciendo y mejorando la construcción de los conceptos que están inmersos en la investigación.
Como preámbulo a cada clase, las docentes proponen emplear la indagación y la elaboración de material didáctico a los estudiantes relacionado con el contenido a desarrollar en 
cada taller. Cada sesión tiene como apertura una actividad de inducción para conectar los saberes previos y el desarrollo de competencias en las dos asignaturas trabajadas en la articulación, siendo protagonistas los estudiantes ya que deben consultar y sustentar ante el grupo, la temática indagada a partir de un ejemplo, luego se presenta el taller para ser desarrollado individualmente.

La exposición de cada niño se apoya en carteles, animales fabricados con material reciclable; además, las docentes incorporaron recursos como escucha de audios con onomatopeyas de animales y ritmos musicales para identificar sonidos empleando el sentido del oído, observación de videos complementarios a las temáticas a abordar y debates en torno a estos, presentaciones apoyadas en títeres, proyección de cápsulas de aprendizaje tomadas de www.colombiaaprende.edu. co referentes a estructuras aritméticas y temática trabajada de Ciencias Naturales, representación esquemática del sistema circulatorio y del corazón empleando diversos materiales; en toda esta didáctica, prima la indagación de datos tanto del área de Ciencias Naturales como de Matemáticas, para afianzar aún más la articulación y el aprendizaje activo.

La cuarta fase (Resultados y productos) permite consolidar los datos y se evidencia aprendizaje de las estructuras aritméticas, reconocimiento del lenguaje matemático y resolución de situaciones problema del contexto, además, apropiación de conceptos propios de los contenidos trabajados de Ciencias Naturales.

\section{Resultados y reflexiones}

Se evidencia en los estudiantes, un avance en el análisis y aplicación de los algoritmos necesarios para dar solución a los problemas; además, identifican lenguaje matemático propio de las estructuras aritméticas, que les permite ir ganando habilidades para enfrentar con éxito situaciones problema de la vida real. En esta perspectiva, se considera que el diseño e implementación de diversos problemas aditivos y multiplicativos contribuyen al desarrollo de procesos de pensamiento y competencias matemáticas. Al emplear la indagación, los estudiantes amplían y profundizan cada vez más los conceptos trabajados en los diferentes talleres, se empodera a los estudiantes para que sean constructores y autores de su conocimiento, identifican los conceptos matemáticos dentro de las Ciencias Naturales, desarrollan competencias científicas, la misma articulación permite que emerjan otros aprendizajes y habilidades como la creatividad, la curiosidad y la observación; además, el trabajo colaborativo facilita que los estudiantes asuman el rol del investigador y no se conformen con lo mínimo, sino que busquen explorar más allá de los contenidos trabajados.

El abordar una investigación como esta donde se reflexiona sobre la naturaleza e implicación didáctica de las estructuras aritméticas, así como el trabajar en forma interdisciplinar, permite reconocer en primera instancia la importancia de fortalecer la formación y actualización de los docentes de la Básica Primaria. El docente de primaria debe enfrentarse 
no solo con la responsabilidad de cimentar las bases de los niños desde las dimensiones que componen el ser humano; sino también, debe hacerse cargo en la mayoría de los casos de la enseñabilidad de todas las áreas del conocimiento propuestas en el currículo escolar contempladas en La ley General de Educación, Ley 115 de 1994, en el artículo 23.

Por otro lado, se evidencian dificultades y vacíos a la hora de hablar y comprender el lenguaje matemático implícito en los documentos emanados por el MEN, tales como: Lineamientos Curriculares, Estándares Básicos de Competencias y Derechos Básicos de Aprendizaje versión 2 (DBA); por esta razón, el conocimiento disciplinar en matemáticas que orienta el educador de la Básica Primaria se enfoca algunas veces en reproducir la forma en que él aprendió y en ocasiones errónea, replicando una y otra vez dichos conocimientos a sus estudiantes. Esta investigación ha permitido interiorizar sobre el marco conceptual de las estructuras aritméticas, reconociendo que no se trata únicamente de enseñar un algoritmo para sumar y restar.

Como proceso de autorreflexión y crítica frente al tema, se plantean algunos interrogantes: ¿Se tienen en cuenta la variedad de situaciones aritméticas para la enseñanza?, ¿Se le da al estudiante el tiempo suficiente para familiarizarse con este lenguaje matemático a través del abordaje de diferentes problemas aditivos y multiplicativos?, ¿Se consideran y abordan las diversas categorías en las cuales se clasifican los problemas aditivos y multiplicativos y que dan sentido a estas operaciones aritméticas?, ¿Conocen los docentes estas categorías de las estructuras aritméticas?, ¿Los docentes emplean la interdisciplinariedad en la enseñanza y aprendizaje de los contenidos matemáticos que orientan? Estos cuestionamientos conllevan a generar cambios y transformaciones en las prácticas de aula de los docentes.

\section{Conclusiones}

Mediante el proceso de implementación de la estrategia didáctica, se logró cumplir con los objetivos propuestos, debido a que se llevaron a cabo cada una de las actividades planeadas, se ampliaron los espacios de trabajo interdisciplinar en las asignaturas de Matemáticas y Ciencias Naturales, el trabajo colectivo de las docentes en la articulación de componentes curriculares empleados en el diseño de los talleres permitió, por primera vez en La Institución Educativa La Independencia, romper esquemas institucionales de ver siempre las materias fragmentadas sin ninguna relación de una con la otra, dando así el primer paso para innovar la didáctica en la enseñanza-aprendizaje de las estructuras aritméticas.

Es importante desarrollar la competencia de la indagación en los estudiantes, puesto que promueve el aprendizaje significativo y enfatiza el cuestionamiento, el análisis de datos y el pensamiento crítico, de acuerdo con los planteamientos hechos por Furman (2012), quien afirma que las secuencias Didácticas empleadas por los docentes 
de Ciencias Naturales basadas en la indagación, en las cuales se emplean los contenidos curriculares de cada grado, son una estrategia de desarrollo fructífero a la hora de transformar las prácticas de enseñanza en el aula.

La investigación acción educativa permitió generar en las docentes, una reflexión pedagógica permanente en torno a las actividades propuestas. Visibilizó los alcances en el proceso de aprendizaje, el desarrollo de los componentes cognitivos, sociales y personales en las diferentes acciones desarrolladas en el aula, así como el análisis discursivo que, tanto las docentes como los estudiantes del grado Tercero, generan y proponen durante el desarrollo de cada clase.

Luego de la aplicación del proyecto, se puede afirmar que validamos su efectividad, ya que al ejecutarlo, los estudiantes lo realizaron con agrado, motivación e interés, como los talleres de intervención se diseñaron para fortalecer las falencias encontradas en el diagnóstico, estos fueron significativos, productivos y motivadores para los estudiantes haciendo ver menos compleja la asignatura de Matemáticas.

\section{Referencias}

Addine, F. (Comp.) (2002). Principios para la dirección del proceso pedagógico. La Habana: Pueblo y Educación.

Carpenter, T., Moser, J., \& Romberg, T. (1982). Adittion and subtraction: A cognitive perspective. Hillsdale, New Jersey. Lawrence Erlbaum.

Castro, E., \& Rico, L. (1995). Estructuras aritméticas elementales y su modelación. España: Síntesis.

Congreso de la República de Colombia. (1994). Ley 115. Ley general de Educación. Bogotá.

Fiallo, J. (2012). ¿Cómo formar un pensamiento interdisciplinario desde la escuela? La Habana: Pueblo y Educación.

Figueredo, E., \& Figueredo, M. (2017). Articulación entre Matemáticas y Ciencias Naturales: una estrategia para aprender estructuras aritméticas. Tunja: Universidad Pedagógica y Tecnológica de Colombia.

Flores, P., Castro, E., \& Fernández, J. (2015). Enseñanza y aprendizaje de las estructuras aritméticas. En P. Flores\& L. Rico, Enseñanza y aprendizaje de las matemáticas en Educación Primaria (pp. 205-229). Madrid- España: Pirámide.

Furman, M. (2012). Orientaciones técnicas para la producción de secuencias didácticas para un desarrollo profesional situado en las áreas de matemáticas y ciencias. Bogotá: Ministerio de Educación Nacional. 
Kemmis, S. (1988). Modelo de Investigación acción. En S. Rodríguez, N. Herraíz, Prieto, Marta, M. Martínez, M. Picazo, . . S. Bernal, Investigación acción (pp. 14-15). Madrid: Universidad Autónoma de Madrid.

MEN. (1998a). Lineamientos Curriculares Ciencias Naturales y Educación Ambiental. Bogotá: Magisterio.

MEN. (1998b). Lineamientos Curriculares Matemáticas. Bogotá: Cooperativa editorial magisterio.

Piaget, J. (1978). Introducción a la Epistemología Genética. I. El Pensamiento Matemático (2a. ed). Buenos Aires: Paidós.

Valverde, G., \& Näslund-Hadley, E. (2010). La condición de la educación matemática $y$ ciencias naturales en América Latina y el Caribe. Washinngton, DC.Banco Interamericano de desarrollo.

Vázquez-Alonso, A., \& Rodríguez-Cruz, A. (2014). Formación del profesoradi en naturaleza de la ciencia mediante investigación-acción. Praxis \& Saber, 5(9), 165 188. https://doi.org/10.19053/22160159.3000

Vergnaud, G. (1986). Psicología do desenvolvimento cognitivo e didactica das matematicas. Un exemplo: as estructuras aditivas. In Analise Psicologica, 1, pp. 75-90

Vergnaud, G. (1988). Multiplicative structures. En J. B. Hiebert, Number concepts and operations in the middle grades (pp. 141-161). Hillsdale: Erlbaum. 